\title{
On highly nonintegrable functions and homogeneous polynomials
}

\author{
by P. Wojtaszczyk (Warszawa)
}

\begin{abstract}
We construct a sequence of homogeneous polynomials on the unit ball $\mathbb{B}_{d}$ in $\mathbb{C}^{d}$ which are big at each point of the unit sphere $\mathbb{S}$. As an application we construct a holomorphic function on $\mathbb{B}_{d}$ which is not integrable with any power on the intersection of $\mathbb{B}_{d}$ with any complex subspace.
\end{abstract}

1. Introduction. Let $\mathbb{S}$ denote the unit sphere in the complex space $\mathbb{C}^{d}$. In the paper [5] a sequence $\left(p_{n}(z)\right)_{n=0}^{\infty}$ of homogenous polynomials in $\mathbb{C}^{d}$ was constructed such that $\left|p_{n}(z)\right| \leq 1$ for all $n$ and all $z \in \mathbb{S}$ and $\int_{\mathbb{S}}\left|p_{n}(z)\right|^{2} d \sigma(z) \geq c>0$ for all $n$. Such polynomials can be used to produce holomorphic functions in $\mathbb{B}_{d}$ (the unit ball of $\mathbb{C}^{d}$ ) with "bad" behaviour on almost all slices (cf. [5], Remark 1.10). The "almost all" restriction is caused by the fact that each $p_{n}(z)$ has zeros on $\mathbb{S}$ (unless $d=1$, which is a trivial case), and to conclude something on all slices one has to control the location of the sets where $p_{n}(z)$ is small. On the other hand, from the function theory point of view it is interesting to have results for all slices (see e.g. [2]). In this note we construct a sequence of homogeneous polynomials which allows us to control behaviour on all slices. Our arguments in this note are modifications of some arguments from [5], [7] and [1]. As an application we construct a holomorphic function in the unit ball $\mathbb{B}_{d}$ which is not integrable with any power on any slice.

The author would like to express his gratitude to Prof. J. Siciak for suggesting this problem.

1.1. Geometric notions. In the complex $d$-dimensional space $\mathbb{C}^{d}$ we will always consider the natural scalar product $\langle\cdot, \cdot\rangle$. On the unit sphere $\mathbb{S}$ we

1991 Mathematics Subject Classification: 32A05, 32A35.

Key words and phrases: homogeneous polynomials, highly nonintegrable holomorphic function.

The author was supported by the KBN grant 2P301004.06. 
will consider a unitarily invariant pseudo-metric $\varrho\left(z_{1}, z_{2}\right)$ defined as

$$
\varrho\left(z_{1}, z_{2}\right):=\sqrt{1-\left|\left\langle z_{1}, z_{2}\right\rangle\right|} .
$$

It is clear that $\varrho\left(z_{1}, z_{2}\right)=0$ if and only if $z_{1}=\lambda z_{2}$ for some $\lambda \in \mathbb{C}$ (and clearly $|\lambda|=1)$. As usual, we denote by $B(z ; r)$ the open ball with center $z$ and radius $r$, i.e.

$$
B\left(z_{0} ; r\right):=\left\{z \in \mathbb{S}: \varrho\left(z_{0}, z\right)<r\right\} .
$$

There is a natural, unitarily invariant (Lebesgue) measure on $\mathbb{S}$. We normalize it so that the measure of the whole sphere $\mathbb{S}$ equals 1 and we denote this measure by $\sigma$. Using (1.4.5) of [4] we easily compute that

$$
\sigma(B(z ; r))=\left(2 r^{2}-r^{4}\right)^{d-1} .
$$

This clearly gives

$$
r^{2 d-2} \leq \sigma(B(z ; r)) \leq 2^{d-1} r^{2 d-2} .
$$

Clearly for small $r$ 's the constant on the right hand side can be made as close to 1 as we wish. A subset $A \subset \mathbb{S}$ is called $\alpha$-separated if $\varrho\left(z_{1}, z_{2}\right)>\alpha$ for all distinct elements $z_{1}$ and $z_{2}$ of $A$. It is clear that for $\alpha>0$ each $\alpha$-separated subset of $\mathbb{S}$ is finite. We will consider maximal $\alpha$-separated sets. We always mean maximal in the sense of inclusion of sets.

2. Some homogeneous polynomials. All homogeneous polynomials of degree $n$ constructed in this paper will have the form

$$
p(z)=\sum_{j=1}^{s}\left\langle z, \zeta_{j}\right\rangle^{n}
$$

for some finite subset $\left\{\zeta_{1}, \ldots, \zeta_{s}\right\}$ of $\mathbb{S}$. In order to be able to control values of the polynomial $p$ we will usually assume that the set $\left\{\zeta_{1}, \ldots, \zeta_{s}\right\}$ is $\alpha$-separated for some $\alpha$. The natural and useful degree of separation for polynomials of degree $n$ is $1 / \sqrt{n}$. We start with two lemmas on separated sets.

Lemma 1. Suppose that $\left\{\zeta_{1}, \ldots, \zeta_{s}\right\}$ is a $C / \sqrt{N}$-separated subset of $\mathbb{S}$. For $z \in \mathbb{S}$ let

$$
A_{k}(z):=\left\{i: \frac{k C}{2 \sqrt{N}} \leq \varrho\left(z, \zeta_{i}\right) \leq \frac{(k+1) C}{2 \sqrt{N}}\right\} .
$$

Then for $k=1,2, \ldots$ the set $A_{k}(z)$ has at most $2^{d-1}(k+2)^{2 d-2}$ elements. The set $A_{0}(z)$ has at most one element. 
Pr o of. The assertion about $A_{0}$ is clear. Since the balls $B\left(\zeta_{j} ; C /(2 \sqrt{N})\right)$ are disjoint and

$$
\bigcup_{i \in A_{k}(z)} B\left(\zeta_{i} ; \frac{C}{2 \sqrt{N}}\right) \subset B\left(z ; \frac{(k+2) C}{2 \sqrt{N}}\right)
$$

we get

$$
\begin{aligned}
\# A_{k}(z) & \leq \#\left\{i: \varrho\left(z, \zeta_{i}\right)<\frac{(k+1) C}{2 \sqrt{N}}\right\} \\
& \leq \frac{\sigma\left(B\left(z ; \frac{(k+2) C}{2 \sqrt{N}}\right)\right)}{\sigma\left(B\left(z ; \frac{C}{2 \sqrt{N}}\right)\right)} \\
& \leq \frac{2^{d-1}\left(\frac{(k+2) C}{2 \sqrt{N}}\right)^{2 d-2}}{\left(\frac{C}{2 \sqrt{N}}\right)^{2 d-2}}=2^{d-1}(k+2)^{2 d-2} .
\end{aligned}
$$

Lemma 2. If $A \subset \mathbb{S}$ is $\alpha / \sqrt{N}$-separated then for each $\beta>\alpha$ there exists an integer $K=K(\alpha, \beta)$ such that $A$ can be partitioned into $K$ disjoint $\beta / \sqrt{N}$-separated sets.

P r o of. Let us select from $A$ a maximal $\beta / \sqrt{N}$-separated subset $A_{1}$. Next from $A \backslash A_{1}$ we select a maximal $\beta / \sqrt{N}$-separated subset $A_{2}$. We continue in this way till we exhaust $A$. Let $A_{s}$ be the last non-empty set in this procedure. Take $\zeta \in A_{s}$. Since $A_{s-1}$ is a maximal $\beta / \sqrt{N}$-separated subset of $A \backslash \bigcup_{j=1}^{s-2} A_{j}$ we see that $\zeta \notin A_{s-1}$, so $B(\zeta ; \beta / \sqrt{N}) \cap A_{s-1} \neq \emptyset$. Analogously $B(\zeta ; \beta / \sqrt{N}) \cap A_{s-2} \neq \emptyset$ etc. So we see that $B(\zeta ; \beta / \sqrt{N})$ contains at least $s$ distinct elements of $A$. Looking at the measures of balls as in Lemma 1 we see that $B\left(\zeta ; \frac{\beta+\alpha / 2}{\sqrt{N}}\right)$ contains $s$ disjoint balls of radius $\alpha /(2 \sqrt{N})$. From (3) we obtain

$$
s\left(\frac{\alpha}{2 \sqrt{N}}\right)^{2 d-2} \leq 2^{d-1}\left(\frac{\beta+\alpha / 2}{\sqrt{N}}\right)^{2 d-2}
$$

so $s \leq 2^{3 d-3}(\beta / \alpha+1 / 2)^{2 d-2}$. This gives the required decomposition.

Now we are ready to state some estimates for polynomials (4).

Proposition 1. There exists a constant $C$ (rather large) such that for all integers $N$ large enough, for each $C / \sqrt{N}$-separated subset $\left\{\zeta_{1}, \ldots, \zeta_{s}\right\}$ of $\mathbb{S}$ and each integer $k$ with $N \leq k \leq 2 N$ the polynomial

$$
p(z):=\sum_{j=1}^{s}\left\langle z, \zeta_{j}\right\rangle^{k}
$$

satisfies

(i) $|p(z)| \leq 2$ for all $z \in \mathbb{S}$, 
(ii) $|p(z)| \geq 0.5$ for each $z \in \mathbb{S}$ such that $\varrho\left(z, \zeta_{j}\right) \leq 1 /(4 \sqrt{N})$ for some $j=1, \ldots, s$.

Proof. Note that if $\varrho\left(z, \zeta_{j}\right) \geq \alpha / \sqrt{N}$ and $N \leq k \leq 2 N$ then

$$
\left|\left\langle z, \zeta_{j}\right\rangle^{k}\right| \leq\left(1-\alpha^{2} / N\right)^{k} \leq e^{-\alpha^{2} k / N} \leq e^{-\alpha^{2}} .
$$

Consider the sets $A_{k}(z)$ defined in Lemma 1. From Lemma 1 we obtain

$$
\begin{aligned}
|p(z)| & \leq \sum_{j=1}^{s}\left|\left\langle z, \zeta_{j}\right\rangle\right|^{k} \leq \sum_{k=0}^{\infty} \sum_{i \in A_{k}(z)}\left|\left\langle z, \zeta_{i}\right\rangle\right|^{k} \\
& \leq 1+\sum_{k=1}^{\infty} e^{-(k C / 2)^{2}} 2^{d-1}(k+2)^{2 d-2}
\end{aligned}
$$

It is clear that we can fix a $C>0.5$ such that

$$
\sum_{k=1}^{\infty} e^{-(k C / 2)^{2}} 2^{d-1}(k+2)^{2 d-2} \leq 0.1 .
$$

Such a choice of $C$ clearly ensures (i).

For a fixed $j$ and $z \in \mathbb{S}$ such that $\varrho\left(z, \zeta_{j}\right)<1 /(4 \sqrt{N})$ we have, for $i \neq j$,

$$
\varrho\left(z, \zeta_{i}\right) \geq \frac{C}{\sqrt{N}}-\frac{1}{4 \sqrt{N}} \geq \frac{1}{4 \sqrt{N}} .
$$

This shows that

$$
\left|\left\langle z, \zeta_{j}\right\rangle^{k}\right| \geq\left(1-\frac{1}{16 N}\right)^{k} \geq\left(1-\frac{1}{16 N}\right)^{2 N}
$$

so for $N$ large enough we have

$$
\left|\left\langle z, \zeta_{j}\right\rangle^{k}\right| \geq(1 / 3)^{1 / 8} \geq 0.87 \text {. }
$$

Analogously to the argument for (i) we see from (6) that

$$
\sum_{i \neq j}\left|\left\langle z, \zeta_{i}\right\rangle^{k}\right| \leq \sum_{k=1}^{\infty} \sum_{i \in A_{k}(z)}\left|\left\langle z, \zeta_{i}\right\rangle^{k}\right| \leq 0.1 .
$$

Since

$$
|p(z)| \geq\left|\left\langle z, \zeta_{j}\right\rangle^{k}\right|-\sum_{i \neq j}\left|\left\langle z, \zeta_{i}\right\rangle^{k}\right|
$$

from (7) and (8) we obtain (ii).

Now we are ready for the main technical result of this note.

THEOREM 1. There exists an integer $k=k(d)$ and a sequence $p_{n}(z)$ of homogeneous polynomials of degree $n$ (for $n$ large enough) such that

(i) $\left|p_{n}(z)\right| \leq 2$ for all $z \in \mathbb{S}$,

(ii) for each $s$ (large enough), $\sum_{n=k s}^{k(s+1)-1}\left|p_{n}(z)\right| \geq 0.5$ for all $z \in \mathbb{S}$. 
Proof. Let $k$ be the integer given by Lemma 2 for $\alpha=0.25$ and $\beta=C$ where $C$ is the constant given by Proposition 1 . For $N=s k$ (and such that the estimate of Proposition 1 holds) fix a maximal $1 /(4 \sqrt{N})$-separated subset $A \subset \mathbb{S}$ and using Lemma 2 divide it into $k$ disjoint $C / \sqrt{N}$-separated subsets $A_{0}, A_{1}, \ldots, A_{k-1}$. For $n=s k+j$ we define

$$
p_{n}(z):=\sum_{\zeta \in A_{j}}\langle z, \zeta\rangle^{n}
$$

From Proposition 1 we infer that $\left|p_{n}(z)\right| \leq 2$ (so (i) holds) and $\left|p_{n}(z)\right| \geq 0.5$ for

$$
z \in \bigcup_{\zeta \in A_{j}} B\left(\zeta ; \frac{1}{4 \sqrt{N}}\right) .
$$

Since $A=\bigcup_{l=0}^{k-1} A_{l}$ is a maximal $1 /(4 \sqrt{N})$-separated subset of $\mathbb{S}$ we infer that

$$
\bigcup_{j=0}^{k-1} \bigcup_{\zeta \in A_{j}} B\left(\zeta ; \frac{1}{4 \sqrt{N}}\right)=\bigcup_{\zeta \in A} B\left(\zeta ; \frac{1}{4 \sqrt{N}}\right)=\mathbb{S} .
$$

This gives (ii).

Remark 1 . The sets $A_{j}$ used in the above proof need not be maximal $C / \sqrt{N}$-separated subsets of $\mathbb{S}$. If we enlarge them to get such subsets, say $\widetilde{A}_{j}$, then there are signs $\varepsilon_{\zeta}^{n}$ such that the polynomials

$$
\widetilde{p}_{n}(z)=\sum_{\zeta \in \tilde{A}_{j}} \varepsilon_{\zeta}^{n}\langle z, \zeta\rangle^{n}
$$

will satisfy

$$
\int_{\mathbb{S}}\left|\widetilde{p}_{n}(z)\right|^{2} d \sigma(z)>c>0
$$

for all $n$ and some $C$. This follows from the arguments following Lemma 2.7 of [5]. Clearly those polynomials will also satisfy (i) and (ii) of Theorem 1.

Remark 2. The possibility of generalizing arguments from [5] to yield results like our Theorem 1 was known to A. B. Aleksandrov. In his paper [1] he states (Theorem 4 ) that there is a $K$ (depending only on the dimension $d$ ) such that for each $n$ there are homogeneous polynomials $p_{n}^{s}(z)$ of degree $n$, where $s=1, \ldots, K$, such that for some constants $C \geq c>0$ we have $C \geq \sum_{s=1}^{K}\left|p_{n}^{s}(z)\right| \geq c>0$ for all $s \in \mathbb{S}$. It is easy to modify our proof of Theorem 1 to get this fact.

3. An application. As an easy application of Theorem 1 let us show the following fact: 
The function

$$
\sum_{n} n^{\ln n} p_{n}(z)=: f(z)
$$

is a holomorphic function in $\mathbb{B}_{d}$ such that for each hyperplane $\Pi \subset \mathbb{C}^{d}$ and any $p>0$,

$$
\int_{\Pi \cap \mathbb{B}_{d}}|f(z)|^{p} d \nu(z)=\infty
$$

where $d \nu$ is the volume measure on $\Pi \cap \mathbb{B}_{d}$.

Since $\left|p_{n}(z)\right| \leq 2|z|^{n}$ and the series $\sum n^{\ln n}|z|^{n}$ converges for $|z|<1$ we see that $f(z)$ is a holomorphic function in $\mathbb{B}_{d}$. Hence we easily see that (9) is equivalent to

$$
\int_{z \in \Pi, 0.5<|z|<1}|f(z)|^{p} d \nu(z)=\infty
$$

Writing (10) in polar coordinates (see e.g. 1.4.3 in [4]) we see that in order to show (9) it suffices to consider complex lines $\Pi$ only. It is also clear that only small $p$ 's matter. Thus we must show that for each $w \in \mathbb{S}$ and each $1>p>0$ the function $g_{w}(\lambda):=f(\lambda w)$ defined for $\lambda \in \mathbb{C}$ and $|\lambda|<1$ satisfies

$$
\int_{|\lambda|<1}\left|g_{w}(\lambda)\right|^{p} d \nu(\lambda)=\infty .
$$

But it is known (cf. [3] or [6]) that if a function $g(\lambda)=\sum_{n=0}^{\infty} a_{n} \lambda^{n}$ on the unit disc satisfies

$$
\int_{|\lambda|<1}|g(z)|^{p} d \nu(\lambda)<\infty
$$

then

$$
\left|a_{n}\right|=o\left(n^{2 / p-1}\right) .
$$

But $g_{w}(\lambda)$ has the power series expansion

$$
g_{w}(\lambda)=\sum_{n} n^{\ln n} p_{n}(w) \lambda^{n}
$$

so we infer from Theorem 1 that (12) does not hold. This shows our claim.

This example improves a bit upon Theorem 1 of [2].

\section{References}

[1] A. B. Aleksandrov, Proper holomorphic maps from the ball into a polydisc, Dokl. Akad. Nauk SSSR 286 (1986), 11-15 (in Russian). 
[2] P. Jakóbczak, Highly nonintegrable functions in the unit ball, Israel J. Math., to appear.

[3] A. Nakamura, F. Ohya and H. Watanabe, On some properties of functions in weighted Bergman spaces, Proc. Fac. Sci. Tokyo Univ. 15 (1979), 33-44.

[4] W. Rudin, Function Theory in the Unit Ball of $\mathbb{C}^{n}$, Springer, New York, 1980.

[5] J. Ryll and P. Wojtaszczyk, On homogeneous polynomials on a complex ball, Trans. Amer. Math. Soc. 276 (1983), 107-116.

[6] S. V. Shvedenko, On the Taylor coefficients of functions from Bergman spaces in the polydisk, Dokl. Akad. Nauk SSSR 283 (1985), 325-328 (in Russian).

[7] P. Wojtaszczyk, On values of homogeneous polynomials in discrete sets of points, Studia Math. 84 (1986), 97-104.

Institute of Mathematics

Warsaw University

Banacha 2

02-097 Warszawa, Poland

E-mail: przemekw@mimuw.edu.pl 